\title{
Improving general health and reducing burnout of nurses in Namibia
}

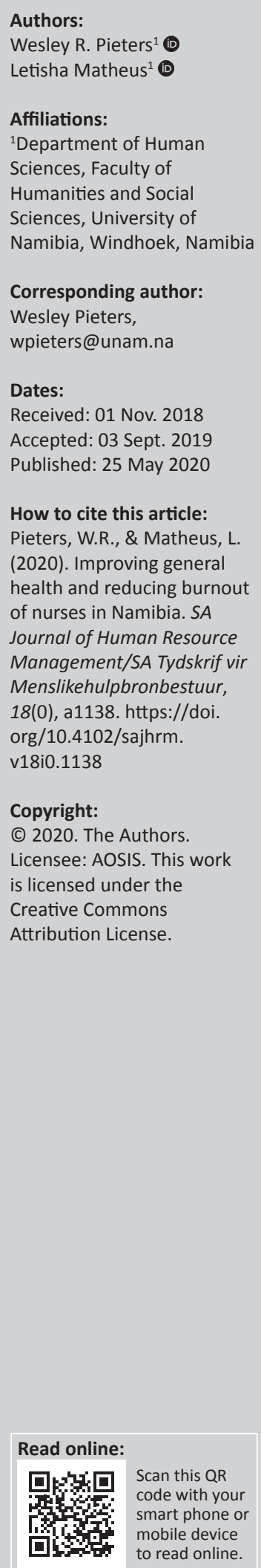

Orientation: Nurses make up $80 \%$ of Namibia's healthcare workforce, and they are considered as the backbone of the industry. Because of the lack of other healthcare providers nurses are exposed to high levels of job stress.

Research purpose: This study investigated how job demands-resources and psychological capital impact general health and burnout of nurses in Oshikoto, Kavango East, Oshana, Omaheke and Khomas regions.

Motivation for the study: Improving the work environment by balancing the relationship between job demands and job resources will result in lower levels of burnout, improved healthcare services, improved employee performance and patient satisfaction.

Research design/approach and method: Using questionnaires, this study investigated the perceptions of nurses within selected regions of Namibia to understand the relationship amongst these variables. The results were analysed using the SPSS (version 24), Pearson's product-moment correlation and multiple regression analyses.

Main findings: Emotional exhaustion was found to have a positive relationship with general health and workload. Social dysfunction and anxiety and insomnia were found to be significant predictors of cynicism. Anxiety and insomnia, workload and social dysfunction were found to be significant predictors of emotional exhaustion.

Practical/managerial implications: Healthcare sector needs to invest in health education and stress management programmes for nurses on how to take care of their own health and emotional well-being. Providing training and development opportunities and coping strategies increases nurses' psychological capital, general health, skills and abilities.

Contribution/value-add: This ground breaking study in Namibia will pave the way for future research regarding the health and well-being of health professionals, add to the already existing knowledge within industrial and organisational psychology and guide interventions to improve the health and well-being.

Keywords: job demands-resources; psychological capital; general health; burnout; industrial psychology.

\section{Introduction}

Burnout is a major problem within the nursing profession. Nurses are faced with high levels of burnout because of the nature of their work compared to other health professionals (Khamisa, Oldenburg, Peltzer, \& Ilic, 2015). Burnout is a result of being exposed to unpleasant stressful work environments for a long period of time (Khamisa et al., 2015). Stress can have a negative influence on the health, well-being and job satisfaction of nurses. Stress may also impact the hospital in terms of absenteeism and poor patient care (Mohajan, 2012). Factors that cause job stress include overtime work as well as irregular working hours. Because of the stressful work environment nurses may experience burnout (Mohajan, 2012). Stress is an inevitable part of the working world; however, some professions are more prone to stress.

Nursing is a profession that experiences more stress compared to other professions, and the main causes of stress include physical demands, management issues, lack of resources and difficulties in balancing home and work responsibilities (American Holistic Nurses Association, 2007). Inadequate staffing of nurses and the high levels of nurse burnout are issues that have become a crisis across the world (Health Education England, 2014). It becomes difficult for hospitals to provide good quality care when they experience staff shortages, especially with a growing population (Health Education England, 2014). 
Recent information on nursing jobs in Namibia indicates that nurses make up the highest number of healthcare workers in the public sector, with an estimated number of 2848 registered nurses and 2043 enrolled nurses compared to 666 medical doctors and 288 pharmacists (World Health Organization, 2002). Nurses therefore make up $80 \%$ of Namibia's healthcare workforce (Ministry of Health and Social Services, 2008). This indicates that the country relies heavily on nurses' performance and the quality of their service to patients.

Factors such as quality patient care and working shift patterns can influence job satisfaction and nurses' intention to leave their job (Aiken et al., 2012). Job satisfaction, in turn, has an effect on burnout (Ogresta, Rusac, \& Zorec, 2008). Working long hours under pressure and having to attend to patients with limited resources available may cause nurses to become less satisfied and may result in them resigning (Khamisa et al., 2015).

According to the Job Demands-Resources model (JD-R), job demands are the characteristics of the job that require continuous effort over a period of time (Demerouti, Bakker, Nachreiner, \& Schaufeli, 2001). Job resources are the characteristics of the job that make it easy to achieve work goals of the healthcare team and the hospital, reduce job demands, or stimulate personal growth through significant work, and bring meaning to the lives of the nurses (Bakker \& Demerouti, 2007). When job resources within the hospital are sufficient, it increases the nurses' motivation to stay committed to their work and limit their intentions to leave (Schaufeli \& Bakker, 2004).

According to Lorenz, Beer, Putz, and Heinitz (2016), psychological capital has a positive relationship with job satisfaction and commitment. When employees experience psychological capital, they are more empowered, less likely to resign and will be less absent during work. Psychological capital may influence job performance, and employees are able to draw upon more resources to pursue goals (Lorenz et al., 2016).

This article discusses key literature to better understand the relationship between job demands, job resources, psychological capital, general health and burnout of nurses in Namibia. It further explains how the study was conducted, presents the findings, discusses the results in relations to key literature and makes some practical recommendations about this study. This article begins with the purpose of the study.

\section{Purpose}

In Namibia, the healthcare industry faces challenges such as staff shortages, work overload and poor general health (Gaomas, 2006). The shortage of nurses prevents the smooth operation of hospital services (Gaomas, 2006).
Nurses experience high levels of burnout because of a high workload and because nurses are always in contact with sick patients, they are easily exposed which can cause workrelated health problems (Wang, Kristopher, Guoping, Burgess, \& Williams, 2003).

Burnout levels range between $10 \%$ and $70 \%$ amongst nurses and have become a common issue amongst healthcare workers, which can affect the quality of care provided to patients (Lyndon, 2016). Some of the factors that lead to burnout in the nursing profession include work-related stress that is caused by high job demands, work overtime, shortage of staff and lack of resources (Khamisa et al., 2015).

The purpose of this study was to investigate how job demands, job resources and psychological capital impact the general health and burnout of nurses in Oshikoto, Okavango East, Oshana, Omaheke and Khomas regions in Namibia. Limited research has been conducted to explore the impact of job demands, job resources, psychological capital on the general health and burnout of nurses in Namibia. This study aimed to add to the understanding of general health and burnout of nurses.

\section{Literature review \\ Burnout}

Because of staff shortages, lack of resources and high workloads, the stress nurses experience could lead to frustration, alcoholism, absenteeism, inadequate patient care and potentially burnout (Continuing Education for Healthcare Professionals, 2014; Mohajan, 2012; Shirey, 2006). Burnout is a result of constant exposure to stress which is caused by factors such as increased job demands and work pressure. Burnout causes nurses to detach from work and become less engaged with patients (Lyndon, 2016; Maslach, Jackson, \& Leiter, 1996). According to Maslach, Schaufeli, and Leiter (2001) burnout is a prolonged response to chronic interpersonal stressors on the job and is characterised by emotional exhaustion, cynicism and inefficacy. Bakker, Demerouti, and Euwema (2005) define burnout as the result of an imbalance between job demands and job resources.

The Multidimensional Theory of Burnout describes burnout in a much broader manner in terms of three components: emotional exhaustion, depersonalisation and reduced personal accomplishment (Maslach \& Jackson, 1986). Emotional exhaustion is what employees experience when they feel drained. Depersonalisation refers to a detached, impersonal response towards recipients of one's service. Reduced personal accomplishment refers to a decline in work productivity, reduced self-efficacy and effectiveness that are associated with the inability to cope with job demands (Maslach, 1998). According to Maslach et al. (2001), research carried out on burnout reports that burnout is strongly related to emotional exhaustion and that emotional exhaustion in simpler terms is the stress experienced by workers. 
The influence of burnout can be understood by the JD-R model. The JD-R model tries to combine two independent variables: job demands, which are described as initiators of a health impairment process, and job resources, which are described as initiators of a motivational process (Demerouti \& Bakker, 2011). The health impairment process proposes that jobs with severe job demands, for example, work overload and emotional demands, exhaust employees, lead to strain and ultimately to health problems (Bakker, Demerouti, \& Schaufeli, 2003). The motivational process suggests that job resources have the potential to motivate individuals and lead to high work engagement, but a lack of resources reduces motivation and leads to cynicism (Demerouti \& Bakker, 2011).

\section{General health}

The general well-being and health of nurses are also related to burnout. Well-being consists of physical and psychological aspects. The availability of staff is affected by a hazardous environment in which nurses may work. Nurses are exposed to substances that can cause various infections. Africa has $1.3 \%$ of the health workforce of the world and $25 \%$ of the disease burden in the world (World Health Organization, 2004). When nurses experience poor well-being and health, they are more susceptible to experience burnout.

Stress can result in depression. Stress can cause sleeping problems and can have physical effects such as headaches, stomach upset and changes in blood pressure (National Institute for Occupational Safety and Health, 2008). This indicates that stress affects employees' physical health and well-being.

In this study, general health will be characterised by somatic symptoms (physical ailments), anxiety and insomnia (problems with sleep) and social dysfunction (poor interaction with others) (Maslach et al., 2001). Nurses may be anxious about the quality of service because the lack of adequate resources (Khamisa et al., 2015) affects the quality of their sleep, which is already hampered by shift work (Aiken et al., 2012). Not being able to sleep well is a major factor in influencing the general health as sleep is one of the best ways for the body to recuperate (Robotham, Chakkalackal, \& Cyhlarova, 2011) and recover from the job demands (Bakker et al., 2003). Work stress influences the smooth operation of hospital services (Gaomas, 2006); this also includes interaction with patients (social dysfunction).

\section{Job demands-resources}

Nurses perform poorly in poor working conditions (Ministry of Health and Social Services, 2008). Overcrowding of the public hospitals, failure of hospital staff to pay attention to avoid harm or errors, and alleged mistreatment of patients are some of the issues discussed in newspapers and public debates regarding the health department (Ministry of Health and Social Services, 2008). Other African countries and the Ministry of Health and Social Services in Namibia have the same mandate, which is to ensure that its health departments are operating well to promote the well-being of the citizens.

The HIV and AIDS epidemic impacts life expectancy, causing the latter to decline in the workforce of 17 out of 48 subSaharan African countries, which in turn resulted in unmanageable workload, affected the quality and productivity of work and also caused more frustration amongst nurses (Sanders, Dovlo, Meeus, \& Lehmann, 2003).

The availability of qualified nurses is low in many countries because the number of nurses who graduate from tertiary institutions is low (Munjanja, Kibuka, \& Dovlo, 2005). Nembwaya (2013) reported that although the University of Namibia has been training healthcare professionals, there remains a high need of nurses in the private and public sectors.

Because of poor working conditions, qualified nurses are leaving state hospitals, preferring to work in the private sector. Out of 104 registered nurses, 65 moved to the private health sector in the country between 2001 and 2004, whilst 39 left the country for the United Kingdom (Gaomas, 2006). Faced with unfavourable working conditions and no opportunities for growth in their careers, nurses thus resign from the public health sector (Gaomas, 2006).

Job demands are aspects of a job that require sustained effort and lead to psychological problems, such as stress and emotional exhaustion. Examples of job demands are work hours, time pressure, work environment and workload (Bakker et al., 2005). They are also further defined in a broader concept of job demand dimensions, which can be grouped into qualitative and quantitative demands. Qualitative demands include role ambiguity and role conflict, and quantitative demands relate to time pressure and workload (Janssen, 2001).

Job demands are not necessarily negative, but excessive effort requirements and exposure to high job demands over a long period of time can lead to burnout (Demerouti et al., 2001). According to Sundin, Hochwälder, and Lisspers (2011), job demands such as time pressure, emotional demands and lack of nursing staff were found to predict both emotional exhaustion and depersonalisation, which are considered to be the core dimensions of burnout. As a result of work-related stress, job demands also have a negative impact on individuals' motivation to engage in work (Bakker, 2010). When employees are required to do much work under time pressure, they may become less engaged.

Job resources are aspects of a job that ease job demands and help employees to succeed in completing work tasks and stimulate personal growth and development (Demerouti et al., 2001). Job resources are also defined as those aspects that foster intrinsic motivation among employees and satisfy the needs for autonomy, belongingness and competence (Hakanen, Bakker, \& Schaufeli, 2006; Van den Broeck, Vansteenkiste, De Witte, \& Lens, 2008). 
Examples of job resources include social support, autonomy and performance feedback (Bakker \& Demerouti, 2007). In this study, job resources include organisational support, job security and advancement opportunities (Jackson \& Rothmann, 2005). Research has shown that strong relationships exist between job resources and work engagement, organisational commitment and job satisfaction (Bakker \& Demerouti, 2007). However, when job demands are high, it can influence work engagement negatively. Previous research has also shown that the lack of job resources may contribute to burnout (Hakanen et al., 2006).

Individuals learn by receiving proper feedback, which increases job competence, whereas support from supervisors and colleagues satisfies the need for autonomy and the need to belong and can help in completing the work faster (Van den Broeck et al., 2008). Job resources facilitate employees' motivation to engage in work and reduce the level of burnout by lessening the impact of job demands (Crawford, Lepine, \& Rich, 2010).

Staff shortages (of nurses) are a serious problem in district hospitals in Namibia. It is reported that district hospitals have complained about staff shortages as a result of the large number of vacant posts (Ministry of Health and Social Services, 1998). It is unlikely to find nurses at the workstations that they were assigned to because these nurses are working alone without any supervision and support from medical officers and registered nurses (Ministry of Health and Social Services, 1998). Most of the qualified staff members do not want to work in the remote areas because of a lack of proper accommodation, work environment and unfair management practices. It is very difficult to recruit qualified staff, and as a result of these challenging work-related factors, nurses do not stay long in these areas (Ministry of Health and Social Services, 1998).

Based on the literature discussed above, the following hypotheses have been developed:

Hypothesis 1: Job demands are positively related to burnout.

Hypothesis 2: Job demands are positively related to general health.

Hypothesis 3: Job resources, organisational support, job security and advancement opportunities are negatively related to burnout.

Hypothesis 4: Job resources, organisational support, job security and advancement opportunities are negatively related to general health.

\section{Psychological capital}

Psychological capital is defined as the positive psychological state of development of an individual, which is characterised by self-efficacy, optimism, hope and resilience (Luthans,
Youseff, \& Avolio, 2007). Self-efficacy refers to a person's confidence in having the ability to complete a challenging task, and it involves five behaviours: high goal-setting, openness to challenging tasks, high self-motivation, application of the necessary effort for goal accomplishment and perseverance. Optimism refers to a person's thinking positively about succeeding now and in the future. Hope refers to a person's motivation to reach goals and create pathways to achieve those goals. Resilience refers to a person's ability to cope and recover from life difficulties and adapt to significant changes (Luthans et al., 2007).

According to Luthans and Church (2002), psychological capital is the study of human strengths and psychological capacities that can be measured, developed and managed for improved employee performance in the workplace.

Self-efficacy, hope, optimism and resilience adopt and maintain a sense of control. When individuals are positive, they will view their chances of being successful high. Having high self-efficacy will give them the motivation to attempt and achieve with challenging tasks. Having hope will encourage different pathways towards reaching their goals. Having resilience will help individuals recover quickly from obstacles (Luthans et al., 2007).

According to Masten, Cutuli, Herbers, and Reed (2009), social support builds self-efficacy and resilience, and resilience builds optimism and hope. When employees receive support from colleagues, it improves their level of self-efficacy; they learn from each other and develop new skills. Developing new skills and abilities improves employees' ability to come with new challenging situations (resilience). When these employees are able to cope better with challenging situations, they become more hopeful and optimistic about the future outcomes.

\section{The relationship between job demands- resources, psychological capital, general health and burnout}

Studies have shown that job demands are positively related to emotional exhaustion and depersonalisation (Maslach \& Jackson, 1981b). When job demands are high, employees experience increased emotional exhaustion, but do not become disengaged; and when job resources are insufficient, employees experience high levels of disengagement but not emotional exhaustion. High job demands and the lack of job resources are the main drivers of burnout (Alarcon, Edwards, \& Menke, 2011; Demerouti et al., 2001).

According to Avey, Wernsing, and Luthans (2008), psychological capital and positive emotions are interrelated. Positive emotions are a result of psychological capital and positivity. Positive emotions can also facilitate and restore physical, social and psychological resources that have been depleted (Fredrickson, 2001). Studies have shown that nurses with a supportive environment and adequate number 
of nursing colleagues are more satisfied with their work, give better quality care and are less likely to experience burnout (Vahey, Aiken, Sloane, Clarke, \& Vargas, 2004).

The United Kingdom has one of the highest rates of nurses reporting burnout across Europe because of a number of risk factors associated with job dissatisfaction and stress. Research studies have confirmed a high level of job satisfaction in less stressful work environments, and about $30 \%$ of nurses have less intention to leave the profession in a healthy work environment (Health Education England, 2014).

A stressful work environment, which is caused by work overload and a shortage of staff, can cause nurses to be dissatisfied with their work. As a result, dissatisfaction amongst nurses may lead to low work engagement, burnout and consequently become a risk to patients. Nurses experienced high levels of job dissatisfaction and job-related stress who work overtime with little support from managers, and less work flexibility and autonomy (Richman, 2006).

According to the JD-R model, the interaction between job demands and job resources is important for the development of strain or motivation (Bakker, Demerouti, Taris, Schaufeli, \& Schreurs, 2003). According to Bakker and Demerouti (2007), the reason why job resources can act as buffers is different for different resources. For example, a quality relationship with one's supervisor can ease the impact of job demands such as work overload, emotional and physical demands on job strain. Support from supervisor may also help the worker to cope with the job demands and act as a protector against ill health (Vaananen, et al., 2003).

The JD-R health model indicates that job demands lead to negative health and job resources lead to positive or negative health. Job resources have a beneficial impact on health, as the more the resources are available, the easier the workers will recover from job demands (Brauchli, Jenny, Fullemann, \& Bauer, 2015). Job resources mediate the relationship between job demands and burnout and general health.

When nurses experience burnout, especially emotional exhaustion, because of high job demands or other stressors, they can protect themselves by mobilising their positive resources as a coping tool (Hobfoll, 1989). A low level of selfefficacy can promote the development of burnout (Cherniss, 1993), and a high level of self-efficacy will result in high levels of engagement and less burnout (Ventura, Salanova, \& Llorens, 2015). According to Laschinger and Fida (2014), psychological capital can protect newly graduated nurses from the occurrence of burnout.

Limited resources, workplace stress, workplace hazards, well-being of nurses and the limited number of nursing staff are the factors that cause major concerns in the

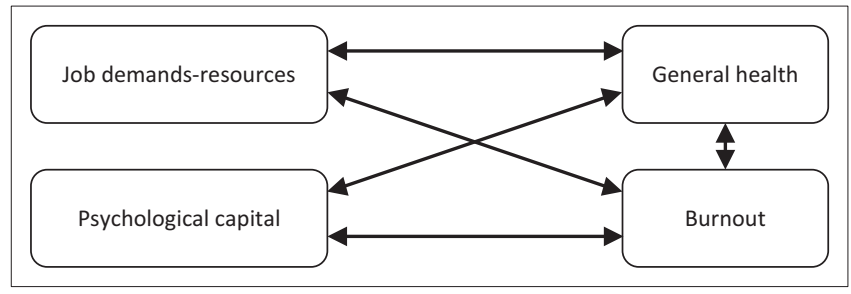

FIGURE 1: Theoretical relationship between job demands-resources, psychological capital, general health and burnout.

health department. The relationship between job resources, job demand and burnout is consistently negative, where the lack of job resources is causing high levels of burnout (Demerouti et al., 2001).

Based on the literature discussed, Figure 1 demonstrates the theoretical relationship between job demands-resources, psychological capital, general health and burnout. Job demands increase the levels of burnout and health (ill health) experienced by employees. Literature also indicates that different job resources may reduce the experience of burnout and general health (ill health). Psychological capital impacts the general health and burnout of employees. Figure 1 indicates that the general health and burnout levels of employees are also interdependent.

Based on the literature discussed, the following hypotheses are proposed:

Hypothesis 5: Psychological capital is negatively related to general health.

Hypothesis 6: Psychological capital is negatively related to burnout.

Hypothesis 7: Job demands (workload) and general health (anxiety and insomnia, somatic symptoms) predict burnout (emotional exhaustion).

Hypothesis 8: General health (anxiety and insomnia, social dysfunction) predicts burnout (cynicism).

Hypothesis 9: Burnout (emotional exhaustion and cynicism) predicts general health (anxiety and insomnia).

Hypothesis 10: Burnout (emotional exhaustion and cynicism) predicts general health (social dysfunction).

\section{Method}

\section{Research approach}

This study used quantitative research in the form of questionnaires to collect data about the biographical information, job demands-resources, psychological capital, general health and burnout of nurses in the selected regions in Namibia. Research assistants were used to collect the data in the different regions where they lived. 


\section{Design}

Permission was obtained from the Permanent Secretary of the Ministry of Health and Social Services, the matrons at the different regional hospitals and station managers within the different hospitals. Once permission was obtained, nurses were informed about their rights to privacy, confidentiality and the purpose of the study. Participants were in no way harmed in this study, and the questionnaires were securely stored for the next 3 years. After informed consent was obtained from the nurses, the questionnaires were distributed to be completed after working hours. In order not to interfere with work-related duties, the questionnaires were collected after a few days as agreed between research assistants and nurses. Participants were informed about their right to withdraw from the study at any time without any negative consequences. A total of 750 questionnaires were distributed within the different regions, of which 672 were completed and returned, providing a response rate of $89.6 \%$.

\section{Participants}

This study used convenience sampling, choosing participants who were willing and available to participate. A sample of 672 nurses included enrolled nurses, registered nurses and student nurses from public hospitals and clinics.

Of the sample of 672 nurses, the majority were women $(n=$ $537,79.9 \%)$. Most of the respondents reported that they are between the ages of 24 and 28 years ( $n=167,24.9 \%$ ), have worked for 3-4 years in the nursing profession $(n=168,25 \%)$, are single ( $n=416,61.9 \%)$, have $1-2$ children ( $n=245,36.5 \%)$, have obtained a certificate $(n=191,28.4 \%)$, are working as a registered nurse $(n=272,40.5 \%)$ and are working in the Khomas region $(n=214,31.8 \%)$. The rest of the biographical information is presented in Table 1.

\section{Measuring instruments}

A biographical questionnaire was developed to collect information about participant's gender, age, tenure, marital status, number of dependants (children), highest qualification obtained, rank and region in which they work.

The Job Demands-Resources Scale (JD-RS) was developed by Jackson and Rothmann (2005) to measure job demands and job resources. The questionnaire made use of a four-point response scale (1: never; 2 : sometimes; 3 : often; and 4: always) and 46 items. The questionnaire consists of five dimensions: workload ('Do you work under pressure?'), resources ('Does your job offer you opportunities for personal growth and development?'), organisational support ('Can you count on your colleagues when you come across difficulties in your work?'), job security ('Do you need to be more secure that you will still be working in one year's time?') and advancement opportunities ('Does your organisation give you opportunities to follow training courses?'). Jackson and Rothmann (2005) found this instrument to be reliable, recording Cronbach's alpha coefficients of 0.75 for workload, 0.76 for resources, 0.88 for organisational support, 0.90
TABLE 1: Frequency distribution of the sample $(n=672)$.

\begin{tabular}{|c|c|c|}
\hline Item & Frequency & Percentage \\
\hline \multicolumn{3}{|l|}{ Gender } \\
\hline Male & 133 & 19.8 \\
\hline Female & 537 & 79.9 \\
\hline Missing values & 2 & 0.3 \\
\hline \multicolumn{3}{|l|}{ Age (year) } \\
\hline Below 24 & 127 & 18.9 \\
\hline $24-28$ & 167 & 24.9 \\
\hline 29-31 & 99 & 14.7 \\
\hline $32-35$ & 71 & 10.6 \\
\hline $36-40$ & 51 & 7.6 \\
\hline $41-45$ & 33 & 4.9 \\
\hline $46-50$ & 37 & 5.5 \\
\hline 51 and older & 83 & 12.4 \\
\hline Missing values & 4 & 0.6 \\
\hline \multicolumn{3}{|l|}{ Tenure (year) } \\
\hline Less than 1 & 68 & 10.1 \\
\hline $1-2$ & 135 & 20.1 \\
\hline $3-4$ & 168 & 25.0 \\
\hline $5-6$ & 57 & 8.5 \\
\hline $7-8$ & 33 & 4.9 \\
\hline $9-10$ & 37 & 5.5 \\
\hline $11-15$ & 38 & 5.7 \\
\hline 16 and more & 131 & 19.5 \\
\hline Missing values & 5 & 0.7 \\
\hline \multicolumn{3}{|l|}{ Marital status } \\
\hline Single & 416 & 61.9 \\
\hline Married & 208 & 31.0 \\
\hline Divorced & 18 & 2.7 \\
\hline Widowed & 27 & 4.0 \\
\hline Missing values & 3 & 0.4 \\
\hline \multicolumn{3}{|c|}{ Number of dependants } \\
\hline None & 216 & 32.1 \\
\hline $1-2$ & 245 & 36.5 \\
\hline $3-4$ & 123 & 18.3 \\
\hline $5-6$ & 52 & 7.7 \\
\hline 7-9 & 15 & 2.2 \\
\hline 10 and more & 18 & 2.7 \\
\hline Missing values & 3 & 0.4 \\
\hline \multicolumn{3}{|l|}{ Qualifications } \\
\hline Grade 12 & 165 & 24.6 \\
\hline Certificate & 191 & 28.4 \\
\hline Diploma & 163 & 24.3 \\
\hline Degree & 62 & 9.2 \\
\hline Honours degree & 80 & 11.9 \\
\hline Master's degree & 4 & 0.6 \\
\hline Missing values & 7 & 1.0 \\
\hline \multicolumn{3}{|l|}{ Rank } \\
\hline Enrolled nurse & 211 & 31.4 \\
\hline Registered nurse & 272 & 40.5 \\
\hline Student nurse & 186 & 27.7 \\
\hline Missing values & 3 & 0.4 \\
\hline \multicolumn{3}{|l|}{ Region } \\
\hline Oshikoto & 150 & 22.3 \\
\hline Kavango East & 152 & 22.6 \\
\hline Oshana & 59 & 8.8 \\
\hline Omaheke & 97 & 14.4 \\
\hline Khomas & 214 & 31.8 \\
\hline Total & 672 & 100 \\
\hline
\end{tabular}

for job security and 0.80 for advancement opportunity. Pieters and Hasheela (2018) found an acceptable reliability statistics in a Namibian study: workload (0.76), resources (0.75), organisational support (0.88), job security (0.79) and advancement opportunities (0.88). 
The Psychological Capital Questionnaire (PsyCapQ) was developed by Luthans and Youssef (2004) to measure the positive psychological state of individuals. This questionnaire consists of a six-point Likert-type scale (1: strongly disagree; 2: disagree; 3: somewhat disagree; 4: somewhat agree; 5: agree; and 6: strongly agree) and 24 items. It consists of four subscales: self-efficacy ('I feel confident analysing a long-term problem to find a solution'), hope ('If I should find myself in a jam at work, I could think of many ways to get out of it'), resilience ('When I have a setback at work, I have trouble recovering from it, moving on') and optimism ('I always look on the bright side of things regarding my job'). This instrument was found to be reliable, recording Cronbach's alpha coefficients of 0.87 for self-efficacy, 0.84 for hope, 0.80 for optimism and 0.86 for resilience (Sapyaprapa, Tuicomepee, \& Watakakosol, 2013).

The General Health Questionnaire (GHQ) was developed by Goldberg (Goldberg \& Hillier, 1979) to detect health problems and the perception of quality of life. There are different versions, the 12, 28, 30 or 60 items of the GHQ (Jackson, 2007). This study made use of the 28 -item version, which consists of three subscales: somatic symptoms ('I have been feeling perfectly well and in good health'), anxiety and insomnia ('I have lost much sleep over worry') and social dysfunction ('I felt that life is entirely hopeless'). The items are measured on a four-point response scale (1: not at all; 2 : no more than usual; 3: rather more than usual; and 4: much more than usual). This instrument was found to be reliable, recording Cronbach's alpha coefficient ranging from 0.78 to 0.95 (Jackson, 2007).

The Maslach Burnout Inventory (MBI) was developed by Maslach and Jackson (1981b) and it measures three domains of burnout: emotional exhaustion, cynicism and professional efficacy. It has a 7-point response scale (0: never; 1 : a few times a year or less; 2 : once a month; 3 : a few times a month; 4: once a week; 5: a few times a week; and 6: every day) with 16 items (Maslach, Jackson, \& Leiter, 1996). Sample items include emotional exhaustion ('I feel emotionally drained from my work'), cynicism ('I doubt the significance of my work') and professional efficacy ('I can effectively solve the problems that arise in my work'). This instrument was found to be reliable, recording Cronbach's alpha coefficients of 0.88 for emotional exhaustion, 0.67 for cynicism and 0.78 for professional efficacy (Bria, Spanu, Baban, \& Dumitrasfu, 2014). Pieters and Van Heerden (2018) found an acceptable reliability for emotional exhaustion. However, cynicism and professional efficacy did not meet the standards for acceptable reliability of 0.70 within a Namibian study.

\section{Analysis}

The IBM SPSS Programme (Version 24) was used to analyse the quantitative data collected from the questionnaires (SPSS, 2016). The results were presented in descriptive statistics (mean, standard deviation and reliability) and inferential statistics (Pearson's correlation coefficient) was used to determine the relationship between the variables.

The reliability of the results was measured using the Cronbach's alpha coefficient of 0.7 , which represents a reliable scale. The Pearson's product-moment correlation was used to determine the relationship between the different variables. The stepwise multiple regression analysis was used to indicate which variable best predicts the dependent variables and allows the researchers to identify which dimensions need to be included in the analysis.

\section{Ethical consideration}

Ethical clearance was awarded by the University of Namibia and permission to conduct the study was provided by the Permanent secretary of the Ministry of Health and Social Services, Namibia. All ethical principles have been upheld in this study (Ref no.: 17/3/3).

\section{Results \\ Descriptive statistics and correlations}

The means (M), standard deviation (SD), Cronbach's alpha coefficient and correlations are recorded in Table 2.

TABLE 2: Mean, standard deviation, Cronbach's alpha and Pearson product-moment correlation.

\begin{tabular}{|c|c|c|c|c|c|c|c|c|c|c|c|c|c|c|c|c|}
\hline \multirow[t]{2}{*}{ Variable } & \multicolumn{2}{|c|}{ Total } & \multirow[t]{2}{*}{$\alpha$} & \multirow[t]{2}{*}{1} & \multirow[t]{2}{*}{2} & \multirow[t]{2}{*}{3} & \multirow[t]{2}{*}{4} & \multirow[t]{2}{*}{5} & \multirow[t]{2}{*}{6} & \multirow[t]{2}{*}{7} & \multirow[t]{2}{*}{8} & \multirow[t]{2}{*}{9} & \multirow[t]{2}{*}{10} & \multirow[t]{2}{*}{11} & \multirow[t]{2}{*}{12} & \multirow[t]{2}{*}{13} \\
\hline & M & SD & & & & & & & & & & & & & & \\
\hline 1. JDR_WL & 23.74 & 4.40 & 0.78 & - & - & - & - & - & - & - & - & - & - & - & - & - \\
\hline 2. JDR_RES & 22.50 & 4.42 & 0.75 & $0.12 *$ & - & - & - & - & - & - & - & - & - & - & - & - \\
\hline 3. JDR_OS & 62.89 & 10.31 & 0.88 & $0.06 *$ & $0.54 * * *$ & - & - & - & - & - & - & - & - & - & - & - \\
\hline 4. JDR_JS & 8.88 & 2.63 & 0.79 & $0.13 *$ & $0.24 *$ & $0.31 * *$ & - & - & - & - & - & - & - & - & - & - \\
\hline 5. JDR_ADV & 11.68 & 4.77 & 0.88 & $-0.06 *$ & $0.32 * *$ & $0.30 * *$ & $0.18^{*}$ & - & - & - & - & - & - & - & - & - \\
\hline 6. PsyCap_SE & 26.18 & 6.07 & 0.83 & -0.00 & $0.26^{*}$ & $0.26 *$ & $0.13 *$ & $0.15^{*}$ & - & - & - & - & - & - & - & - \\
\hline 7. PsyCap_H & 27.67 & 5.76 & 0.84 & -0.01 & $0.26 *$ & $0.23 *$ & $0.12 *$ & $0.06 *$ & $0.65 * * *$ & - & - & - & - & - & - & - \\
\hline 8. PsyCap_R & 17.04 & 4.07 & 0.70 & $0.06^{*}$ & $0.16^{*}$ & $0.06 *$ & $0.05 *$ & $-0.06 *$ & $0.42 * *$ & $0.53^{* * *}$ & - & - & - & - & - & - \\
\hline 9. GH_SS & 12.53 & 4.55 & 0.82 & $0.27 *$ & $-0.07 *$ & $-0.15 *$ & 0.02 & $-0.12 *$ & $-.08^{*}$ & $-0.07 *$ & 0.02 & - & - & - & - & - \\
\hline 10. GH_Al & 12.11 & 5.16 & 0.89 & $0.27 *$ & $-0.14 *$ & $-0.20 *$ & -0.00 & $-0.06 *$ & $-0.14 *$ & $-0.14 *$ & -0.02 & $0.65 * * *$ & - & - & - & - \\
\hline 11. GH_SD & 22.07 & 6.16 & 0.84 & $0.20 *$ & $-0.08^{*}$ & $-0.16^{*}$ & -0.03 & $0.02 *$ & $-0.12 *$ & $-0.17 *$ & $-0.08 *$ & $0.50 * * *$ & $0.60 * * *$ & - & - & - \\
\hline 12. BO_EXH & 13.83 & 9.69 & 0.88 & $0.41 * *$ & $-0.15^{*}$ & $-0.18 *$ & 0.01 & $-0.04 *$ & $-0.14 *$ & $-0.15^{*}$ & -0.02 & $0.39 * *$ & $0.49 * *$ & $0.39 * *$ & - & - \\
\hline 13. BO_CYN & 5.75 & 5.55 & 0.75 & $0.26 *$ & $-0.08^{*}$ & $-0.12 *$ & 0.02 & $0.12 *$ & $-0.07 *$ & $-0.12 *$ & -0.04 & $0.25 *$ & $0.38 * *$ & $0.40 * *$ & $0.53 * * *$ & - \\
\hline
\end{tabular}

JD-R, job demands-resources; JDR_WL, job demands-resources (workload); JDR_RES, job demands-resources (resources); JDR_OS, job demands-resources (organisational support); JDR_JS, job demands-resources (job security); JDR_ADV, job demands-resources (advancement opportunities); PsyCap, psychological capital; PsyCap_SE, psychological capital (self-efficacy); PsyCap_H, psychological capital (hope); PsyCap R, psychological capital (resilience); GH, general health; GH_SS, general health (somatic symptoms); GH_Al, general health (anxiety and insomnia); GH_SD, general health (social dysfunction); BO, burnout; BO_EXH, burnout (emotional exhaustion); BO_CYN, burnout (cynicism).

$*$, Statistically significant: $p \leq 0.05$; **, Practically significant correlation (medium effect): $0.30 \leq r \leq 0.49$; ***, Practically significant correlation (large effect): $r \geq 0.50$. 
The JD-RS questionnaire was found to be reliable in this study. A Cronbach's alpha coefficient of 0.78 was reported for workload, 0.75 for resources, 0.88 for organisational support, 0.79 for job security and 0.88 for advancement opportunities.

The PsyCapQ was found to be reliable in this study. A Cronbach's alpha coefficient of 0.83 was reported for selfefficacy, 0.84 for hope and 0.70 for resilience. Optimism was found to be unreliable; thus, it was not included further in the analysis as it did not meet the Cronbach's alpha coefficient of 0.70 .

The GHQ was found to be reliable in this study. A Cronbach's alpha coefficient of 0.82 was reported for somatic symptoms, 0.89 for anxiety and insomnia and 0.84 for social dysfunction.

The Maslach Burnout Inventory was found to be reliable in this study, reporting with a Cronbach's alpha coefficient of 0.88 for emotional exhaustion and 0.75 for cynicism. Professional efficacy was found to be unreliable and thus excluded from further analysis in this study because it did not meet the Cronbach's alpha coefficient of 0.70 .

Workload reported a mean of 23.74 and a standard deviation of 4.40; resources reported a mean of 22.50 and a standard deviation of 4.42; organisational support reported a mean of 62.89 and a standard deviation of 10.31 ; job security reported a mean of 8.88 and a standard deviation of 2.63; and advancement opportunity reported a mean of 11.68 and a standard deviation of 4.77 .

Self-efficacy reported a mean of 26.18 and a standard deviation of 6.07; hope reported a mean of 27.67 and a standard deviation of 5.76; and resilience reported a mean of 17.04 and a standard deviation of 4.07. Somatic symptoms reported a mean of 12.53 and a standard deviation of 4.55 ; anxiety and insomnia reported a mean of 12.11 and a standard deviation of 5.16; and social dysfunction reported a mean of 22.07 and a standard deviation of 6.16. Emotional exhaustion reported a mean of 13.83 and a standard deviation of 9.69; cynicism reported a mean of 5.75 and a standard deviation of 5.55 .

Making use of Pearson's correlation coefficient, Table 2 shows that workload reported a positive relationship with resources $(r=0.12, p<0.05$; small effect), organisational support $(r=$ $0.06, p<0.05$; large effect) and job security $(r=0.13, p<0.05$; small effect), and a negative relationship with advancement opportunity $(r=-0.06, p<0.05$; small effect). Workload reported a negative relationship with self-efficacy $(r=-0.00$, $p<0.05$; small effect), a negative relationship with hope $(r=$ -0.01, $p<0.05$; small effect) and a positive relationship with resilience ( $r=0.06, p<0.05$; small effect). Workload reported a positive relationship with somatic symptoms $(r=0.27, p<$ 0.05 ; small effect), a positive relationship with anxiety and insomnia ( $r=0.27, p<0.05$; small effect) and a positive relationship with social dysfunction $(r=0.20, p<0.05$; small effect). Table 2 indicates that workload reported a positive relationship with emotional exhaustion $(r=0.41, p<0.05$; medium effect) and a positive relationship with cynicism $(r=0.26, p<0.05 ;$ small effect $)$.

Resources reported a positive relationship with organisational support $(r=0.54, p<0.05$; large effect), job security $(r=0.24$, $p<0.05$; small effect) and advancement opportunity $(r=0.32$, $p<0.05$; medium effect). Resources reported a positive relationship with self-efficacy ( $r=0.26, p<0.05$; small effect), a positive relationship with hope $(r=0.26, p<0.05$; small effect) and a positive relationship with resilience $(r=0.16$, $p<0.05$; small effect). Resources reported a negative relationship with somatic symptoms $(r=-0.07, p<0.05$; small effect), a negative relationship with anxiety and insomnia $(r=-0.14, p<0.05$; small effect) and a negative relationship with social dysfunction ( $r=-0.08, p<0.05$; small effect). Resources reported a negative relationship with emotional exhaustion ( $r=-0.15, p<0.05$; small effect) and a negative relationship with cynicism ( $r=-0.08, p<0.05$; small effect).

Organisational support reported a positive relationship with job security $(r=0.31, p<0.05$; medium effect $)$ and advancement opportunities ( $r=0.30, p<0.05$; small effect). Organisational support reported a positive relationship with self-efficacy $(r=0.26, p<0.05$; small effect), a positive relationship with hope ( $r=0.23, p<0.05$; small effect) and a positive relationship with resilience $(r=0.06, p<0.05$; small effect). Organisational support reported a negative relationship with somatic symptoms $(r=-0.15, p<0.05$; small effect), a negative relationship with anxiety and insomnia ( $r=-0.20, p<0.05$; small effect) and a negative relationship with social dysfunction $(r=-0.16, p<0.05$; small effect). Organisational support reported a negative relationship with emotional exhaustion $(r=-0.18, p<0.05$; small effect) and a negative relationship with cynicism $(r=$ $-0.12, p<0.05$; small effect).

Job security reported a positive relationship with advancement opportunity ( $r=0.18, p<0.05$; small effect). Job security reported a positive relationship with self-efficacy $(r=0.13, p<0.05$; small effect), a positive relationship with hope $(r=0.12, p<0.05$; small effect $)$ and a positive relationship with resilience $(r=0.05, p<0.05$; small effect $)$. Job security reported a positive relationship with somatic symptoms ( $r=0.02, p<0.05$; small effect), a negative relationship with anxiety and insomnia $(r=-0.00, p<0.05$; small effect) and a negative relationship with social dysfunction $(r=-0.03, p<0.05$; small effect). Job security reported a positive relationship with emotional exhaustion $(r$ $=0.01, p<0.05$; small effect $)$ and a positive relationship with cynicism $(r=0.02, p<0.05$; small effect).

Advancement opportunity reported a positive relationship with self-efficacy ( $r=0.15, p<0.05$; small effect), a positive relationship with hope $(r=0.06, p<0.05$; small effect $)$ and a negative relationship with resilience $(r=-0.06, p<0.05$; small effect). Advancement opportunity reported a negative relationship with somatic symptoms $(r=-0.12, p<0.05$; 
small effect), a negative relationship with anxiety and insomnia ( $r=-0.06, p<0.05$; small effect) and a positive relationship with social dysfunction $(r=0.02, p<0.05$; large effect). Advancement opportunity reported a negative relationship with emotional exhaustion $(r=-0.04, p<0.05$; small effect) and a positive relationship with cynicism $(r=$ $0.12, p<0.05 ;$ small effect).

Self-efficacy reported a positive relationship with hope $(r=0.65, p<0.05$; large effect) and resilience $(r=0.42$, $p<0.05$, medium effect). Self-efficacy reported a negative relationship with somatic symptoms $(r=-0.08, p<0.05$; small effect), a negative relationship with anxiety and insomnia $(r=-0.14, p<0.05$; small effect) and a negative relationship with social dysfunction $(r=-0.12, p<0.05$; small effect). Self-efficacy reported a negative relationship with emotional exhaustion $(r=-0.14, p<0.05$; small effect) and a negative relationship with cynicism $(r=-0.07, p<$ 0.05; small effect).

Hope reported a positive relationship with resilience $(r=0.53$, $p<0.05$; large effect). Hope reported a negative relationship with somatic symptoms ( $r=-0.07, p<0.05$; small effect), a negative relationship with anxiety and insomnia $(r=-0.14$, $p<0.05$; small effect) and a negative relationship with social dysfunction $(r=-0.17, p<0.05$; small effect). Hope reported a negative relationship with emotional exhaustion $(r=-0.15$, $p<0.05$; small effect) and a negative relationship with cynicism $(r=-0.12, p<0.05 ;$ small effect).

Resilience reported a positive relationship with somatic symptoms ( $r=0.02, p<0.05$; small effect), a negative relationship with anxiety and insomnia $(r=-0.02, p<0.05$; small effect) and a negative relationship with social dysfunction ( $r=-0.08, p<0.05$; small effect). Resilience reported a negative relationship with emotional exhaustion $(r=-0.02, p<0.05$; small effect $)$ and a negative relationship with cynicism $(r=-0.04, p<0.05$; small effect).

Somatic symptoms reported a positive relationship with anxiety and insomnia $(r=0.65, p<0.05$; large effect) and social dysfunction ( $r=0.50, p<0.05$, large effect). Somatic symptoms reported a positive relationship with emotional exhaustion ( $r=0.39, p<0.05$; medium effect) and a positive relationship with cynicism $(r=0.25, p<0.05$; small effect).
Anxiety and insomnia reported a positive relationship with social dysfunction ( $r=0.60, p<0.05$; large effect). Anxiety and insomnia also reported a positive relationship with emotional exhaustion $(r=0.49, p<0.05$; medium effect $)$ and a positive relationship with cynicism $(r=0.38, p<0.05$; medium effect).

Social dysfunction reported a positive relationship with emotional exhaustion ( $r=0.39, p<0.05$; medium effect) and a positive relationship with cynicism $(r=0.40, p<0.05$; medium effect).

Emotional exhaustion reported a positive relationship with cynicism $(r=0.53, p<0.05$; large effect).

\section{Multiple regression analyses}

Multiple regression analysis was performed to test hypotheses 7, 8, 9 and 10 . Some dimensions of the variables were excluded from the analysis because these dimensions did not meet the expected correlation of $r=0.3$ (Pallant, 2005). To test Hypothesis 7, anxiety and insomnia, somatic symptoms and social dysfunction (general health) were used as the independent variables in the first step and workload (job demands) was added in the second step to predict emotional exhaustion (burnout), the dependent variable. The results are reported in Table 3.

A stepwise multiple regression analysis was performed to evaluate how well anxiety and insomnia, somatic symptoms and social dysfunction (general health), and workload (job demands) predict emotional exhaustion (burnout). Table 2 indicates that anxiety and insomnia, somatic symptoms and social dysfunction (general health) produced a significant model in step $1\left(\mathrm{~F}_{(3,604)}=71.21 ; p<0.00\right)$ and accounted for $26 \%$ of the variance. In step 2, workload (job demands) was added to the model, producing a significant model $\left(\mathrm{F}_{(4,603)}=\right.$ 77.85; $p<0.00)$, accounting for $34 \%$ of the variance. The results showed that anxiety and insomnia $(\beta=0.31 ; t=6.41$; $p<0.00)$ is the strongest significant predictor of emotional exhaustion, somatic symptoms $(\beta=0.05 ; t=1.14 ; p<0.26)$ was an insignificant predictor of emotional exhaustion and social dysfunction $(\beta=0.13 ; t=3.11 ; p<0.00)$ was a significant predictor of emotional exhaustion. Workload $(\beta=0.29 ; t=$ 8.51; $p<0.00)$ was also a significant predictor of emotional

TABLE 3: Multiple regression with emotional exhaustion (burnout) being the dependent variable and anxiety and insomnia, somatic symptoms and social dysfunction (general health), and workload (job demands) being the independent variables.

\begin{tabular}{|c|c|c|c|c|c|c|c|c|}
\hline \multirow[t]{2}{*}{ Model } & \multicolumn{2}{|c|}{ Unstandardised coefficients } & \multirow{2}{*}{$\begin{array}{l}\text { Standardised coefficients } \\
\qquad(\beta)\end{array}$} & \multirow[t]{2}{*}{$t$} & \multirow[t]{2}{*}{$p$} & \multirow[t]{2}{*}{$F$} & \multirow[t]{2}{*}{$R^{2}$} & \multirow[t]{2}{*}{$\Delta R^{2}$} \\
\hline & $B$ & SE & & & & & & \\
\hline 1 & - & - & - & - & 0.00 & 71.21 & 0.26 & 0.26 \\
\hline 2 & - & - & - & - & 0.00 & 77.85 & 0.34 & 0.34 \\
\hline (Constant) & -14.45 & 1.98 & - & -7.34 & 0.00 & - & - & - \\
\hline GH_A/I & 0.57 & 0.09 & 0.31 & 6.41 & $0.00 *$ & - & - & - \\
\hline GH_SS & 0.11 & 0.10 & 0.05 & 1.14 & 0.26 & - & - & - \\
\hline GH_SD & 0.21 & 0.07 & 0.13 & 3.11 & $0.00 *$ & - & - & - \\
\hline JDR_WL & 0.65 & 0.08 & 0.29 & 8.51 & $0.00 *$ & - & - & - \\
\hline
\end{tabular}

$t$, test; $p$, probability value; $F$, overall significance; $R^{2}$, percentage variance explained; $\Delta R^{2}$, change in percentage variance explained; $\beta$, standardised coefficient; B, unstandardised coefficients; SE, standard error; GH_SS, general health (somatic symptoms); GH_Al, general health (anxiety and insomnia); GH_SD, general health (social dysfunction); JDR_WL, job demands-resources (workload). $*, p \leq 0.01$. 
TABLE 4: Multiple regression analysis with cynicism (burnout) being the dependent variable and anxiety and insomnia and social dysfunction (general health) being the independent variables.

\begin{tabular}{|c|c|c|c|c|c|c|c|c|}
\hline \multirow[t]{2}{*}{ Model } & \multicolumn{2}{|c|}{ Unstandardised coefficients } & \multirow{2}{*}{$\begin{array}{l}\text { Standardised coefficients } \\
\text { ( } \beta \text { ) }\end{array}$} & \multirow[t]{2}{*}{$t$} & \multirow[t]{2}{*}{$p$} & \multirow[t]{2}{*}{$F$} & \multirow[t]{2}{*}{$R^{2}$} & \multirow[t]{2}{*}{$\Delta \boldsymbol{R}^{2}$} \\
\hline & $B$ & SE & & & & & & \\
\hline (Constant) & -2.39 & 0.74 & - & -3.23 & 0.00 & 74.58 & 0.19 & 0.19 \\
\hline GH_A/I & 0.26 & 0.05 & 0.24 & 5.38 & $0.00 *$ & - & - & - \\
\hline GH_SD & 0.23 & 0.04 & 0.25 & 3.63 & $0.00 *$ & - & - & - \\
\hline
\end{tabular}

$t$, test; $p$, probability value; $F$, overall significance; $R^{2}$, percentage variance explained; $\Delta R^{2}$, change in percentage variance explained; $\beta$, standardised coefficient; $\mathrm{B}$, unstandardised coefficients; $\mathrm{SE}$, standard error; GH_Al, general health (anxiety and insomnia); GH_SD, general health (social dysfunction).

$*, p \leq 0.01$.

TABLE 5: Multiple regression with anxiety and insomnia (general health) being the dependent variable and emotional exhaustion and cynicism (burnout) being the dependent variables.

\begin{tabular}{|c|c|c|c|c|c|c|c|c|}
\hline \multirow[t]{2}{*}{ Model } & \multicolumn{2}{|c|}{ Unstandardised coefficients } & \multirow{2}{*}{$\begin{array}{l}\text { Standardised coefficients } \\
\qquad(\beta)\end{array}$} & \multirow[t]{2}{*}{$t$} & \multirow[t]{2}{*}{$p$} & \multirow[t]{2}{*}{$F$} & \multirow[t]{2}{*}{$R^{2}$} & \multirow[t]{2}{*}{$\Delta R^{2}$} \\
\hline & $B$ & SE & & & & & & \\
\hline (Constant) & 8.18 & 0.31 & - & 26.34 & 0.00 & 117.50 & 0.27 & 0.27 \\
\hline BO_EXH & 0.22 & 0.02 & 0.41 & 10.30 & $0.00 *$ & - & - & - \\
\hline BO_CYN & 0.16 & 0.04 & 0.17 & 4.30 & $0.00 *$ & - & - & - \\
\hline
\end{tabular}

$t$, test; $p$, probability value; $F$, overall significance; $R^{2}$, percentage variance explained; $\Delta R^{2}$, change in percentage variance explained; $\beta$, standardised coefficient; $\mathrm{B}$, unstandardised coefficients; $\mathrm{SE}$, standard error; BO_EXH, burnout (emotional exhaustion); BO_CYN, burnout (cynicism).

$*, p \leq 0.01$.

TABLE 6: Multiple regression with social dysfunction (general health) as the dependent variable and emotional exhaustion and cynicism (burnout) as the independent variables.

\begin{tabular}{|c|c|c|c|c|c|c|c|c|}
\hline \multirow[t]{2}{*}{ Model } & \multicolumn{2}{|c|}{ Unstandardised coefficients } & \multirow{2}{*}{$\begin{array}{l}\text { Standardised coefficients } \\
\qquad(\beta)\end{array}$} & \multirow[t]{2}{*}{$t$} & \multirow[t]{2}{*}{$p$} & \multirow[t]{2}{*}{$F$} & \multirow[t]{2}{*}{$R^{2}$} & \multirow[t]{2}{*}{$\Delta R^{2}$} \\
\hline & $B$ & SE & & & & & & \\
\hline (Constant) & 18.19 & 0.39 & - & 46.75 & 0.00 & 79.85 & 0.20 & 0.20 \\
\hline BO_EXH & 0.16 & 0.03 & 0.25 & 5.98 & $0.00 *$ & - & - & - \\
\hline BO CYN & 0.29 & 0.05 & 0.26 & 6.28 & $0.00 *$ & - & - & - \\
\hline
\end{tabular}

$t$, test; $p$, probability value; $F$, overall significance; $R^{2}$, percentage variance explained; $\Delta R^{2}$, change in percentage variance explained; $\beta$, standardised coefficient; $B$, unstandardised coefficients; $\mathrm{SE}$, standard error; BO_EXH, burnout (emotional exhaustion); BO_CYN, burnout (cynicism).

$*, p \leq 0.01$.

exhaustion and the second largest significant predictor in this model.

To test Hypothesis 8, anxiety and insomnia and social dysfunction (general health) were used as the independent variables in the first step to predict cynicism (burnout), the dependent variable. The results are reported in Table 4.

A stepwise multiple regression analysis was performed to evaluate how well anxiety and insomnia and social dysfunction (general health) predict cynicism (Hypothesis 8). Table 4 produced a significant model in step $1\left(\mathrm{~F}_{(2,628)}=74.58\right.$; $p<0.00)$ that accounted for $19 \%$ of the variance. The results showed that anxiety and insomnia $(\beta=0.24 ; t=5.38 ; p<0.00)$ was a significant predictor of emotional exhaustion and social dysfunction $(\beta=0.25 ; t=5.63 ; p<0.00)$ was the strongest significant predictor of emotional exhaustion (by very small margin).

Emotional exhaustion and cynicism (burnout) were used as the independent variables to predict anxiety and insomnia (general health), the dependent variable (Hypothesis 9). Emotional exhaustion and cynicism were entered into the first model and the results are presented in Table 5.

A stepwise multiple regression analysis was carried out to evaluate how well emotional exhaustion and cynicism predict anxiety and insomnia. Table 5 produced a significant model in step $1\left(\mathrm{~F}_{(2,643)}=117.50 ; p<0.00\right)$ and accounted for $27 \%$ of the variance. The results showed that emotional exhaustion ( $\beta=0.41 ; t=10.30 ; p<0.00$ ) was the strongest significant predictor of anxiety and insomnia and cynicism $(\beta=0.17 ; t=4.30 ; p<0.00)$ was also a significant predictor of anxiety and insomnia.

Emotional exhaustion and cynicism (burnout) were used as the independent variables to predict social dysfunction (general health), the dependent variable (Hypothesis 10). Emotional exhaustion and cynicism were entered into the first model and the results are presented in Table 6 .

A stepwise multiple regression analysis was performed to evaluate how well emotional exhaustion and cynicism predict social dysfunction. In Table 6 emotional exhaustion and cynicism produced a significant model in step $1\left(\mathrm{~F}_{(2,633)}=\right.$ $79.85 ; p<0.00)$ and accounted for $20 \%$ of the variance in social dysfunction (general health). The results showed that emotional exhaustion $(\beta=0.25 ; t=5.98 ; p<0.00)$ was a significant predictor of social dysfunction and cynicism $(\beta=$ $0.26 ; t=6.28 ; p<0.00$ ) was the strongest significant predictor of social dysfunction (by a small margin).

\section{Discussion}

\section{Outline of results}

This study found that job demands had a positive relationship with burnout (emotional exhaustion and cynicism), supporting Hypothesis 1 of this study. The results support the findings of Bakker et al. (2005), Maslach and Jackson (1981b) and Demerouti et al. (2001), which indicated that 
when nurses are expected to perform many tasks as part of their duties, they may experience emotional exhaustion and cynicism.

This study also hypothesised that job demands are positively related to general health. Workload reported a positive relationship with anxiety and insomnia, somatic symptoms and social dysfunction, supporting Hypothesis 2 of this study. A heavy workload negatively impacts the general health of nurses, making them anxious, experience insomnia and physical problems, and become hopeless about life. The results of this study supported hypotheses 3 and 4, indicating that when employees have better access to job resources, organisational support, job security and advancement opportunities, they may become less exhausted, less negative about their abilities at work, less anxious, sleep better at night and develop a positive outlook on life. Similar results were also found in other studies (Bakker, Demerouti, \& Schaufeli, 2003; Bakker \& Demerouti, 2007; Vaananen et al., 2003). This study found a positive relationship between advancement opportunities (job resources) and cynicism (burnout). This is different from what Bakker et al. (2003) found, reporting that job resources ease the impact of job demands. When employees are promoted, they are likely to be required to take on more tasks or more challenging tasks. It was also noted that as a result of a lack of resources and high job demands, employees are likely to experience burnout (Alarcon, Edwards, \& Menke, 2011; Demerouti et al., 2001). Bakker et al. (2003) indicated that the interaction between job demands and job resources can result in either motivation or strain. Job resources can motivate employees to become engaged in their work, whilst a lack of resources can lead to cynicism (Demerouti \& Bakker, 2011). Based on this reasoning, it could be seen that the resources (advancement opportunities) within the work environment are not adequate to motivate employees and rather cause strain.

When employees are more confident about their own abilities, more optimistic about work goals and able to handle stressful situations at work, they experience less physical ailments, are able to sleep good at night and also become calmer and optimistic about life. These findings support Hypothesis 5 regarding the negative relationship between psychological capital and general health.

This study also supports Hypothesis 6 and found that psychological capital (self-efficacy, hope and resilience) had a negative relationship with burnout (emotional exhaustion and cynicism). These findings support the study by Ventura et al. (2015) and Laschinger and Fida (2014) that indicate that low self-efficacy can result in burnout and high self-efficacy can result in increased engagement and reduced burnout.

Workload was found to be a significant predictor of emotional exhaustion. This study found somatic symptoms, anxiety and insomnia and social dysfunction to be significant predictors of emotional exhaustion, supporting Hypothesis 7. Anxiety and insomnia and social dysfunction were found to be a significant predictor of cynicism, supporting
Hypothesis 8. These findings support the study by Bakker and Demerouti (2007), indicating that chronic job requirements such as work overload and emotional demands exhaust employees' mental and physical resources and may therefore lead to the depletion of energy (i.e. a state of emotional exhaustion) and to health problems. This indicates that when nurses do not get enough sleep and their health status is low, they tend to experience high levels of emotional exhaustion, which lead to poor patient care and result in nurses being cynical about their work.

This study found that emotional exhaustion and cynicism predicted anxiety and insomnia as well as social dysfunction, supporting hypotheses 9 and 10. The National Institute for Occupational Safety and Health (2008) highlights that stress can result in depression, may cause sleeping problems and may have a physical impact on nurses, such as headaches, stomach upset and changes in blood pressure.

\section{Practical implications}

This study recommends teamwork and support from colleagues and supervisors as a way to reduce job demands and stimulate personal growth through significant work; this would bring meaning to the lives of the nurses. Job resources such as social support, autonomy and performance feedback can reduce burnout. Individuals learn by receiving proper feedback, which increases job competence, whereas support from supervisors and colleagues satisfies the need for autonomy and the need to belong and can help in completing the work faster (Van den Broeck et al., 2008).

Seeing that some nurses have only completed Grade 12, a certificate or diploma, it is suggested to provide nonaccredited training or short courses that will enhance the capabilities and competences of nurses. These short courses will add to job resources, thereby allowing better adaptation with job demands, improved hope and optimism, enhanced positive general health and reduced levels of burnout. Recruitment and training of learners who completed Grade 12 could help to alleviate the immediate pressure that nurses experience.

It is important for nurses to have good health and wellbeing so that they would be able to provide quality care to patients and also would be satisfied with their work. This study further recommends that the Ministry of Health and Social Services needs to implement training and development programmes and invest in health education and stress management programmes for nurses. It is suggested that nurses should also invest in hobbies or other activities, such as hiking or meditating, to help reduce workrelated stresses.

When nurses are more informed about and actively engaged in improving their own health, they would be better equipped and eager to attend to clients. Once customer service improves, providing quality healthcare becomes possible. 
This, in turn, will increase the optimism and outlook of nurses, productivity and the positive public image of the nursing profession. Once nurses live a healthy and active life, they would also have more personal resources, buffering the job demands, and be less likely to experience burnout.

This study also provides theoretical insights into the relationship of job demands, job resources, psychological capital and how these variables impact general health and burnout specifically for nurses in Namibia. It also provides scientific evidences about the reliability of these instruments and how the findings add to the knowledge within the field of human resource management and industrial psychology.

\section{Limitations}

This study has the following limitations. Firstly, as the study only focused on selected regions within Namibia, the findings are applicable to the regions under study and thus cannot be generalisable to all settings. Secondly, it used a survey design which could have been influenced by response bias.

\section{Recommendations}

It is suggested that future studies should consider a quantitative approach to investigate further approaches to reduce poor health and burnout experienced by nurses in Namibia. Perhaps future studies could also investigate the state of all the healthcare professionals across the country. A longitudinal study or a study focusing on other factors that may impact burnout and general health, which were not included in this study, may be conducted in the future.

\section{Conclusion}

When sufficient job resources are available within the hospital, it increases nurses' motivation to stay engaged in their work and limits their intentions to leave by lessening the influence of job demands (Schaufeli \& Bakker, 2004). It is suggested that adequate resources should be provided to reduce high workload, which in turn results in lowering the levels of anxiety, insomnia and social dysfunction.

The availability of job resources facilitates employees' motivation to engage in work and reduces the level of burnout by lessening the impact of job demands (Crawford et al., 2010). Employees who are required to execute a high workload are likely to experience poor health and ultimately experience burnout. The impact of burnout may be dampened when employees are provided with the required job resources.

General health and burnout levels of nurses are inter-related. The working conditions may negatively impact the burnout levels of nurses, which in turn affects the general health, depleting personal resources and exacerbating the effects of burnout.

\section{Acknowledgements}

The authors would like to thank N. Auanga, J. Kadhila, I. Mwila, N. Nobukoshi and M. Pinehas, the research assistants, for the data collection and their tireless efforts. They would also like to express their gratitude to the employees within the Ministry of Health and Social Services for their accommodating and generous nature with which they engaged in this study.

\section{Authors' contributions}

W.P. conducted the analysis of the data and wrote the article. L.M. is a research student who was involved in the collection of data, as well as in the writing of this article.

\section{Competing interests}

The authors have declared that no competing interests exist.

\section{Funding information}

This research received no specific grant from any funding agency in the public, commercial or not-for-profit sectors.

\section{Data availability statement}

Data sharing is not applicable to this article as no new data were created or analyzed in this study.

\section{Disclaimer}

The views and opinions expressed in this article are those of the authors and do not necessarily reflect the official policy or position of any affiliated agency of the authors.

\section{References}

Aiken, L.H., Sermeus, W., Van den Heede, K., Sloane, D.M., Busse, R., McKee, M., ... Kutney-Lee, A. (2012). Patient safety, satisfaction, and quality of hospital care: Cross sectional surveys of nurses and patients in 12 countries in Europe and the United States. British Medical Journal, 344,7851. https://doi.org/10.1136/bmj. United States. British Medical Journal, 344,7851. https://doi.org/10.1136/bmj.
e1717

Alarcon, G.M., Edwards, J.M., \& Menke, L.E. (2011). Student burnout and engagement A test of the conservation of resources theory. Journal of Psychology, 145(3), 211-227. https://doi.org/10.1080/00223980.2011.555432

American Holistic Nurses Association. (2007). Holistic nursing: Scope and standards of practice. Silver Spring, MD: Nursebooks.org, The Publishing Program of ANA.

Avey, J.B., Wernsing, T.S., \& Luthans, F. (2008). Can positive employees help positive organizational change? Journal of Applied Behavioral Science, 44, 48-70. https://doi. org/10.1177/0021886307311470

Bakker, A.B. (2010). Engagement and job crafting: Engaged employees create their own place to work. In Albrecht, S.L. (Ed.), Handbook of employee engagement: Perspectives, issues, research and practice (pp. 229-244).

Bakker, A.B., \& Demerouti, E. (2007). The job demands-resources model: State of the art. Journal of Managerial Psychology, 22, 309-328. https://doi. org/10.1108/02683940710733115

Bakker, A.B., Demerouti, E., \& Euwema, M.C. (2005). Job resources buffer the impact of job demands on burnout. Journal of Occupational Health Psychology, 10, 170-180. https://doi.org/10.1037/1076-8998.10.2.170

Bakker, A.B., Demerouti, E., \& Schaufeli, W.B. (2003). Dual processes at work in a call centre: An application of the job demands - Resources model. European Journal of Work and Organizational Psychology, 12, 393-417. https://doi. org/10.1080/13594320344000165

Bakker, A.B., Demerouti, E., Taris, T., Schaufeli, W.B., \& Schreurs, P. (2003). A multi-group analysis of the job demands-resources model in four home care organizations. International Journal of Stress Management, 10, 16-38. https:// doi.org/10.1037/1072-5245.10.1.16 
Brauchli, R., Jenny, G.J., Fullemann, D., \& Bauer, G.F. (2015). Towards a job demands resources health model: Empirical testing with generalizable indicators of job demands, job resources, and comprehensive health outcomes. BioMed Research International, 2015, 1-12. https://doi.org/10.1155/2015/959621

Bria, M., Spanu, F., Baban, A., \& Dumitrascu, D.L. (2014). Maslach burnout inventorygeneral survey: Factorial validity and invariance among Romanian healthcare professionals. Burnout Research, 1(3), 103-111. https://doi.org/10.1016/j. professionals. Burno
burn.2014.09.001

Cherniss, C. (1993). Role of professional self-efficacy in the etiology and amelioration of burnout. In Schaufeli, W.B., Maslach, C., \& Marek, T. (Eds.), Series in applied psychology: Social issues and questions. Professional burnout: Recent applied psychology: Social issues and questions. Profer
developments in theory and research (pp. 135-149).

Continuing Education for Healthcare Professionals. (2014). Burnout: Impact on nursing US. Retrieved from http://www.netce.com/coursecontent.php?courseid=827.

Crawford, E.R., Le Pine, J.A., \& Rich, B.L. (2010). Linking job demands and resources to employee engagement and burnout: $A$ theoretical extension and meta-analytic test. Journal of Applied Psychology, 95, 834-848. https://doi.org/10.1037/ a0019364

Demerouti, E., \& Bakker, A.B. (2011). The job demands-resources model: Challenges for future research. South African Journal of Industrial Psychology, 37(2), 974-1038. https://doi.org/10.4102/sajip.v37i2.974

Demerouti, E., Bakker, A.B., Nachreiner, F., \& Schaufeli, W.B. (2001). The job demandsresources model of burnout. Journal of Applied Psychology, 86, 499-512. https:// doi.org/10.4102/sajip.v37i2.974

Fredrickson, B.L. (2001). The role of positive emotions in positive psychology: The broaden and-build theory of positive emotions. American Psychologist, 56(3), 218-226. https://doi.org/10.1037/0003-066X.56.3.218

Gaomas, S. (2006, March 13). Nurses are overworked. New Era Newspaper Namibia. Archived News.

Goldberg, D.P., \& Hillier, V.F. (1979). A scaled version of the general health questionnaire Psychological Medicine, 9, 139-145. https://doi.org/10.1037/0003-066X.56.3.218

Hakanen, J.J., Bakker, A.B., \& Schaufeli, W.B. (2006). Burnout and work engagement among teachers. Journal of School Psychology, 43, 495-513. https://doi. org/10.1016/j.jsp.2005.11.001

Health Education England. (2014). Growing nursing numbers: Literature review on nurses leaving the NHS.

Hobfoll, S.E. (1989). Conservation of resources. A new attempt at conceptualizing stress. American Psychologist, 44(3), 513-524. https://doi.org/10.1037//0003 066X.44.3.513

Jackson, C. (2007). The General Health Questionnaire. Journal of Occupationa Medicine, 57, 79. https://doi.org/10.1093/occmed/kql169

Jackson, L.T.B., \& Rothmann, S. (2005). Work-related well-being of educators in a district of the North West Province. Perspectives in Education, 23, 107-122.

Janssen, O. (2001). Fairness perception as a moderator in the curvilinear relationships between job demands and job resources, satisfaction and job performance. Academy of Management Journal, 44, 1039-1050. https://doi. performance. Academ
org/10.2307/3069447

Khamisa, N., Oldenburg, B., Peltzer, K., \& Ilic, D. (2015). Work related stress, burnout, job satisfaction and general health of nurses. International Journal of Environmental Research and Public Health, 12, 652-666. https://doi.org/10.3390/ ijerph120100652

Laschinger, H.K.S., \& Fida, R. (2014). New nurses burnout and workplace wellbeing: the influence of authentic leadership and psychological capital. Burnout Research 1, 19-28. http://doi.org/10.1016/j.burn.2014.03.002

Lorenz, T., Beer, C., \& Heinitza, K. (2016). Measuring psychological capital: Construction and validation of the compound PsyCap scale (CPC-12). PLoS One, 11(4), 1-17. https://doi.org/10.1371/journal.pone.0152892

Luthans, F., \& Church, A. (2002). Positive organizational behavior: Developing and managing psychological strengths. Academy of Management, 16, 57-75. https:// doi.org/10.5465/ame.2002.6640181

Luthans, F., Youssef, C.M., \& Avolio, B.J. (2007). Psychological capital. New York: Oxford University Press. https://doi.org/10.1037/t06483-000

Lyndon, A. (2016). Burnout amongst health professionals and its effect on patient safety. Agency for Healthcare Research and Quality, 1, 1-17.

Maslach, C. (1998). A multidimensional theory of burnout. In Cooper, C.L. (Ed.), Theories of organisational stress (pp. 68-85). New York: Oxford University Press.

Maslach, C., \& Jackson, S.E. (1981a). Maslach burnout inventory. Palo Alto, CA: Consulting Psychologists Press.

Maslach, C., \& Jackson, S.E. (1981b). The measurement of experiences burnout Journal of Occupational Behavior, 2, 99-113. https://doi.org/10.1037/t05190-000

Maslach, C., \& Jackson, S.E. (1986). Maslach burnout inventory. Palo Alto, CA: Consulting Psychologists Press.

Maslach, C., Jackson, S.E., \& Leiter, M.P. (1996). Maslach burnout inventory manual (3rd edn.). Palo Alto, CA: Consulting Psychologists Press.

Maslach, C., Schaufeli, W.B., \& Leiter, M.P. (2001). Job burnout. Annual Review of Psychology, 52, 397-422. https://doi.org/10.1146/annurev.psych.52.1.397
Masten, A.S., Cutuli, J.J., Herbers, J.E., \& Reed, M.G.J. (2009). Resilience in development. Oxford Handbooks online.

Ministry of Health and Social Services (MOHSS). (1998). Towards achieving health and social wellbeing for all Namibians: A policy framework. Windhoek: Ministry of Health and Social Services.

Ministry of Health and Social Services (MOHSS). (2008). Health and social services review. Namibia: Ministry of Health and Social Services.

Mohajan, H.K. (2012). The occupational stress and risk of it among the employees. International Journal of Mainstream Social Science, 2(2), 17-34.

Munjanja, O., Kibuka, S., \& Dovlo, D. (2005). The Nursing Workforce in Sub-Saharan Africa. ICN 2005 Issue 7: The Global Nursing Review Initiative.

National Institute for Occupational Safety and Health (NIOSH). (2008). Exposure to stress, occupational hazards in hospitals. Department of Health and Human Services, Centers for Disease Control and Prevention, National Institute for Occupational Safety and Health, DHHS (NIOSH) Publication No. 2008-2136.

Nembwaya, H. (2013, May 17). Nam faces shortages of nurses. The Namibian newspaper. Retrieved from https://www.namibian.com.na/index.php?id=108182\&page=archiveread.

Ogresta, J., Rusac, S., \& Zorec, L. (2008). Relation between burnout syndrome and job satisfaction among mental health workers. Croatian Medical Journal, 49(3), 364-374. https://doi.org/10.3325/cmj.2008.3.364

Pallant, J. (2005). SPSS survival manual: A step by step guide to data analysis using SPSS for Windows (version 12). Sydney: Ligare.

Pieters, W.R., \& Hasheela, C. (2018). Investigating the exhaustion of police offices, selected regions within Namibia. Journal of Economics and Behavioral Studies, 10(3), 8499. https://doi.org/10.22610/jebs.v10i3.2319

Pieters, W.R., \& Van Heerden, A.A. (2018). Investigating the relationship between workload-resources and exhaustion of nurses and police officers in Namibia. Journal of Economics and Behavioral Studies, 10(5), 195-207. https://doi. org/10.22610/jebs.v10i5.2509

Richman, A. (2006). Everyone wants an engaged workforce: How can you create it? Workspan, 36-39.

Robotham, D., Chakkalackal, L., \& Cyhlarova, E. (2011). Sleep matters: The impact of sleep on health and wellbeing. Mental Health Foundation Retrieved from https://www.mentalhealth.org.uk/sites/default/files/MHF-SleepReport-2011.pdf

Sanders, D., Dovlo, D., Meeus, W., \& Lehmann, U. (2003). Public health in Africa. In Beaglehole, R. (Ed.), Global public health: A new era. New York: Oxford University Press.

Sapyaprapa, S., Tuicomepee, A., \& Watakosol, R. (2012). Validation of psychological capital questionnaire in Thai employees. The Asian Conference on Psychology and the Behavioral Sciences Official Conference Proceedings, 394-399.

Schaufeli, W.B., \& Bakker, A.B. (2004). Job demands, job resources, and their relationship with burnout and engagement: A multi-sample study. Journal of Organizational Behavior, 25, 293-315. https://doi.org/10.1002/job.248

Shirey, M.R. (2006). Authentic leaders creating healthy work environments for nursing practice. American Journal of Critical Care, 1(3), 256-267.

SPSS (2016). SPSS 24.0 for Windows. Chicago, IL: SPSS Incorporated.

Sundin, L., Hochwälder, J., \& Lisspers, J. (2011). A longitudinal examination of generic and occupational specific job demands and work-related social support associated with burnout among nurses in Sweden. Work, 38, 389-400.

Vaananen, A., Toppinen-Tanner, S., Kalimo, R., Mutanen, P., Vahtera, J., \& Peiro, J.M. (2003). Job characteristics, physical and psychological symptoms, and social support as antecedents of sickness absence among men and women in the private industrial sector. Social Science and Medicine, 57, 807-824. https://doi. org/10.1016/S0277-9536(02)00450-1

Vahey, D.C., Aiken, L.H., Sloane, D.M., Clarke, S.P., \& Vargas, D. (2004). Nurse burnout and patient satisfaction. Medical Care, 42(2), 57-66. https://doi.org/10.1097/01 mlr.0000109126.50398.5a

Van den Broeck, A., Vansteenkiste, M., De Witte, H., \& Lens, W. (2008). Explaining the relationships between job characteristics, burnout, and engagement: The role of basic psychological need satisfaction. Work \& Stress, 22, 277-294. https://doi. org/10.1080/02678370802393672

Ventura, M., Salanova, M., \& Ilorens, S. (2015). Professional self-efficacy as a predictor of burnout and engagement: the role of challenge and hindrance demands. The Journal of Psychology, 149(3), 277-302. https://doi.org/10.1080/ 00223980.2013.876380

Wang, H., Kristopher, F., Guoping, H., Burgess, J., \& Williams, A.B. (2003). A training programme for prevention of occupational exposure to blood-borne pathogens: Impact on knowledge, behaviour and incidence of needle stick injuries among student nurses in Changsha, People's Republic of China. Journal of Advanced Nursing, 41, 187-195. https://doi.org/10.1046/j.13652648.2003.02519.x

World Health Organization (WHO). (2002). Nursing and midwifery services. Strategic directions 2002-2008. Geneva: World Health Organization.

World Health Organization (WHO). (2004). Addressing Africa's health workforce crisis: An avenue for action the high level forum on the health MDGs. Abuja December 2004. Geneva, Switzerland: WHO. 